\title{
MODELING PERFORMANCE INDICATORS' SELECTION PROCESS FOR VO PARTNERS' SUGGESTIONS
}

\author{
Fabiano Baldo ${ }^{1}$, Ricardo J. Rabelo ${ }^{1}$, Rolando V. Vallejos ${ }^{2}$ \\ ${ }^{I}$ Federal University of Santa Catarina, BRAZIL \\ baldo@gsigma.ufsc.br,rabelo@das.ufsc.br \\ ${ }^{2}$ University of Caxias do Sul, BRAZIL \\ rvvallej@ucs.br
}

\begin{abstract}
In the current dynamic world where organizations are inserted in, there is no time to postpone businesses due to the lack of support that helps them to establish the required connections with other organizations agilely. In this context, one of the critical issues is the creation of Virtual Organization, when partners have to be selected. This paper addresses a very closely related issue, which is the selection of the performance indicators that should be applied to drive the partners search. Considering the complexity of this task, this paper presents the process modeling designed to support the user to find appropriated performance indicators that can be used to compare and to suggest organizations that are able to fulfill a business' requirements.
\end{abstract}

\section{INTRODUCTION}

Globalization has been imposing an increasing competition among companies. Looking at customers, lower costs, higher quality and shorter delivery dates keep being essential elements in the competitive matrix of companies. However, this selection is a complex problem. Part of the problem is related to the intrinsic nature of Collaborative Networked Organizations (CNO), where companies are disperse and heterogeneous at several dimensions. In more volatile alliances, like Virtual Organizations (VO), this problem is more difficult as the usual processes of making quotations and selecting partners should be carried out even faster.

In this work, a VO is seen as a dynamic, temporary and logical aggregation of autonomous organizations that cooperate with each other to attend a given collaboration opportunity $(\mathrm{CO})$ or to cope with a specific need, where partners share risks, costs and benefits, and whose operation is achieved via a coordinated sharing of skills, resources and information, enabled by computer networks (Rabelo et al. , 2004). In this context, the right selection of suppliers/partners is simply crucial for the effective success of a VO as a wrong election can impact negatively the competitive matrix of the VO as a whole.

However, alliances of type VOs have other particularities that make this aforementioned task very hard as, per definition, a VO is something unique, i.e. partners can't be always the same even for similar COs, as it happens in traditional supply chains. A CO comes from different customers and countries, which have different cultures, different regulations, different standards, and that apply different metrics in terms of e.g. quality, manufacturing processes and environmental cares. Therefore, the search for partners that can attend all the specificities of a given VO must also involve the selection 
of the most suitable performance indicators (PI) that companies should respect in order to become real candidates / members of that VO. It is important to highlight that PI selection phase precedes the classical Partners Search and Suggestion/Selection phases within the VO Creation framework.

Actually, companies' profiles use to be expressed in the form of PIs. This also uses to be taken into account for internal benchmarking purposes of a $\operatorname{VBE}^{1}$ (Vallejos et al. , 2006). The problem is that managers use to make the selection of PIs without any methodological support or computer assistance. In practice, this means that the selected PIs are almost the same for any CO despite the (usual) hundreds or even dozens of PIs existing in a CNOs' Performance Measurement System (PMS). On the other hand, and even due to the enormous disparity in the PIs' definitions, terminologies and meaningfulness, managers don't have time to go through PMS to analyze PIs one by one. Instead, with the hurry of the business life, they get used to pick the same set of PIs. Worse than this, in the medium-long terms, this affects the VBE quality as partners don't use to improve the PIs associated to their internal processes if they are not forced to.

As a contribution to this problem, this paper presents a process modeling for PI selection as well as the rationale of a supporting system being developed for that. The advantage of such modeling is twofold. Firstly, it helps in the understanding of this process, leaving perfectly clear which are the inputs, outputs, resources and triggers of every involved activity, the interdependences, as well as the correct sequence of activations. Secondly, it can be used for automation this process, including the information integration requirements.

This paper is organized as follows. In section 2, the problem of finding criteria for VO partners' suggestion is depicted. In section 3, the strategy of selecting criteria to suggest partners is shown. Section 4 presents the process modeling conceived to identify the most suitable set of criteria. Section 5 addresses a case study. Preliminary assessment and future works are presented at the end.

\section{CRITERIA FOR PARTNER SUGGESTION}

Until few years ago it was supposed that VO partners could be quickly and easily identified and selected from the open universe of existing companies. However, nowadays it is known that this is not as simple as it seems to be (Camarinha-Matos et al. , 2005), and that selection is more feasible and effective when carried out within the scope of CNOs of VBE type, considering that VBEs works with known enterprises and it normally has a set of common PIs settled when they are created.

One idea superseded during the last years is that costs and dates would be enough to suggest VO partners. Today it is a common sense that is necessary to use more meaningful criteria to accurately suggest appropriated organizations as VO partners (Crispim and Sousa, 2007, Jarimo and Pulkkinen, 2005). Petersen (2003) emphasizes the importance to suggest partners using well-defined set of criteria based on common attributes known by every interested organization. Following Petersen's idea, PIs can be used as part of these common set of criteria for partners' suggestion. Some authors are already applying such approach, e.g. Grudzewski et al. (2005) and Seifert et al. (2005). That is an approach that can be used by organizations that use a common PMS, e.g. organizations that belong to VBEs. Nevertheless, most of the PMS are composed of plenty of PIs, like the ones offered by the SCOR model ${ }^{2}$. Höbig (2002) overcomes this problem using generic and high-level PIs provided by PMS. However, to use always the same generic PIs to compare and select VO partners seem not to be useful in practice. So, it is necessary to find a way to select just the relevant ones for each specific CO. Although PMS is an area of research with several available results, a review in the literature has 
revealed the inexistence of any work which tries to systemize and to give a methodological support for the process of PIs selection.

In the proposed approach, the strategy adopted to select the set of relevant PIs is to filter them according to the CO's requirements. This can be seen as a problem of information matching / information retrieval because the only meaningful information in a PI is its own definition. Although being a good approach, it is too simplistic for the tackled problem. PIs are described with different words by each organization, sometimes a different PI represents the same or equivalent PI's underlying concept, and sometimes they have small but relevant differences in their scopes. Yet, they are configured in different PMS according to companies' daily jargon, which in turn not even follow standards terminology (e.g. SCOR, APICS). In other words, PIs are implemented in a different way from companies to companies (Bittencourt and Rabelo, 2005). Another aspect is that CNO requires an additional category of PIs that so far hasn't been considered, like the cooperation level, trustworthiness, and performance history in past VOs (Westphal et al. , 2007).

Therefore, in order to cope with this, an additional level of information is necessary to be introduced. A way to do that is introducing semantics, which can be done by means of semantic annotation using a common ontologies and mappings (Baldo et al. , 2007). Semantic annotation is currently considered the state-of-the-art when information retrieval is concerned. The term annotation refers to the use of auxiliary symbols that are used to modify the interpretation of other symbols (Dorado and Izquierdo, 2003). Semantic annotation techniques use ontologies to perform the proper annotation of the significant words included in the text (Kiryakov et al. , 2004).

\section{PROBLEM FORMALIZATION}

This section formalizes the problem of PI selection to a specific CO. This is made using sets theory, as follows.

Let's assume $P=\{1, \ldots, p\}$ as the set of PIs being used to measure performance in a VBE. Let also assume $R=\{1, \ldots, r\}$ as the set of requirements that should be fulfilled in order to create a VO to satisfy a specific CO. So, in order to figure out whether a PI $i \quad P$ is related to a requirement $j R$, the function $f$ (presents the intersection's cardinality of each PI and each requirement set of relevant terms.

$$
f(i, j)=\left|P d_{i} \cap P r_{j}\right|, \quad \forall i \in P \wedge \forall j \prime
$$

$P d_{i}=$ set of relevant terms presented in the definition of a PI $i \quad P$.

$\mathrm{Pr}_{j}=$ set of relevant terms presented in the description of a requirement $j \quad R$.

A relevant term can be considered every word or sequence of words that contribute to the meaning of either PI definition or requirement description. Examples of relevant terms are substantives, nouns, verbs and adjectives.

In order to introduce more expressivity and meaningfulness in the result of equation 1, it was introduced in conjunction with relevant terms the notion of semantics. This was accomplished through the utilization of semantic annotations and an ontology designed specially to characterize the concepts related to PIs and COs as well as the relationship among them. Now, the relevant terms of both PIs and COs definitions can also be linked to a formal representation, stated in the ontology, using semantic annotations. This additional formalization enables the relevant terms to be semantic relevant terms. Both ontology and semantic relevant terms compose the so called PIs and COs knowledge base.

Let's define $K=\{1, \ldots, k\}$, that is the set of semantic relevant terms included into the PIs and COs knowledge base. 
Due to the introduction of semantics in the PIs selection process, now a PI can also be selected for a specific $\mathrm{CO}$ requirement whether it has semantic relevant terms in common with a requirement $j \quad R$.

Thus, $\quad g$ ( presents the intersection's cardinality of each PI and each requirement set of semantic relevant terms.

$$
g(i, j)=\left|S d_{i} \cap S r_{j}\right|, \quad \forall i \in P \wedge \forall j \mid
$$

$S d_{i}=$ set of semantic relevant terms in a PI $i \quad P$ found in the knowledge base $K$. It is presented in equation 3.

$S r_{j}=$ set of semantic relevant terms in a CO requirement $j \quad R$ found in the knowledge base $K$. It is presented in equation 4 .

$$
\begin{array}{ll}
S d_{i}=\left\{k_{z} \mid P d_{i, y}=k_{z},\right. & \forall i \in P \wedge \forall y \in P d_{i} \wedge \forall z \in \\
S r_{j}=\left\{k_{z} \mid P r_{j, y}=k_{z},\right. & \forall j \in R \wedge \forall y \in P r_{j} \wedge \forall z \in
\end{array}
$$

The objective function to find the PI that have more relevant terms and semantic relevant terms in common for a specific $\mathrm{CO}$ requirement is:

$Z=\max \sum_{i=1}^{p} \sum_{j=1}^{r}(f(i, j)+g(i, j))$

\section{PROCESS MODELING}

The process of selecting PIs for VO partners' suggestion presented in this paper is composed of two macro activities. The first one is carried just once and it is called preparation phase (A1). It comprises the ontology and knowledge base creation as well as the PIs' description semantic annotation. The second one is executed whenever a new VO needs to be created, and it is called operation phase (A2). It performs the acquisition of $\mathrm{CO}$ requirements and the selection of proper PIs. Figure 1 presents this process, modeled in IDEF0 $^{3}$. In rough terms, IDEF0 is a diagram used to model processes from the functional point of view. Each process is modeled as a box, and its main components are: inputs (left), outputs (right), mechanisms (bottom) and enablers (top). Both preparation and operation phases' descriptions and diagrams are shown in Figure 2 and Figure 3, respectively.

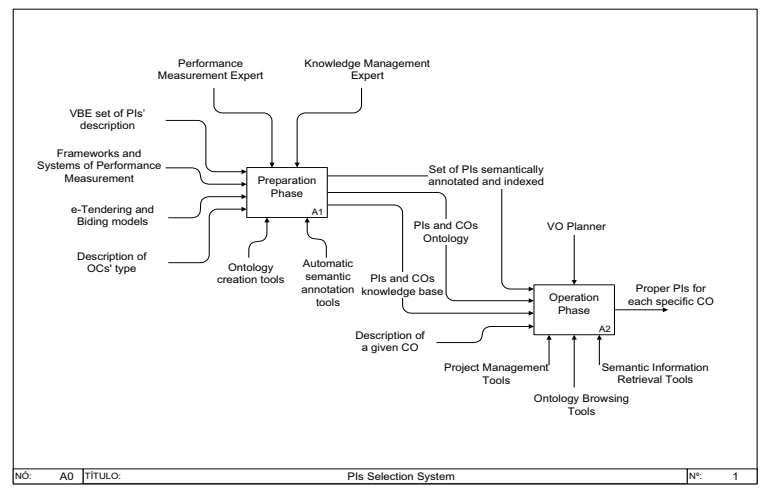

Figure 1. IDEF0 of Performance Indicators Selection Process 
Preparation phase:

A1.1 PIs and COs Ontology Creation: This activity creates the PIs and COs ontology having literature on performance measurement literature and existing eprocurement standardization as the basis for. This formalization is driven by a knowledge management and an expert on PIs.

A1.2 PIs and COs Knowledge Population: This activity performs the population of PIs and COs knowledge base using information from catalogs of PIs as well as from description of $\mathrm{CO}$ types.

A1.3 Automatic PIs Semantic Annotation: In this activity it is applied an automatic semantic annotation technique, combined with PIs and COs ontology and knowledge base, to create annotations in PIs' information.

\section{Operation phase:}

A2.1 CO Requirements Acquisition: it is devoted to the identification of the three main pieces of information that are necessary to make the PIs selection: CO objectives, $\mathrm{CO}$ type and $\mathrm{CO}$ performance requirements.

A2.2 Search Criteria Identification: based on A2.1, the link between CO's information and COs' knowledge base is established in order to identify the semantic terms that will be applied in the semantic information retrieval.

A2.3 PIs Search: This activity performs the semantic information retrieval using the semantic terms provided by the previous activity. The list of PIs that match such semantic terms is generated as the output.

A2.4 Result Analysis and Evaluation: In this activity the result is analyzed and evaluated. This is done to ensure which PIs are suitable to select VO partners. This is a subjective task and it is driven by the user. Regarding its difficulty, support systems can be used to mitigate the subjectivity and to maximize the quality of the results. Even though, an expert can refine this.

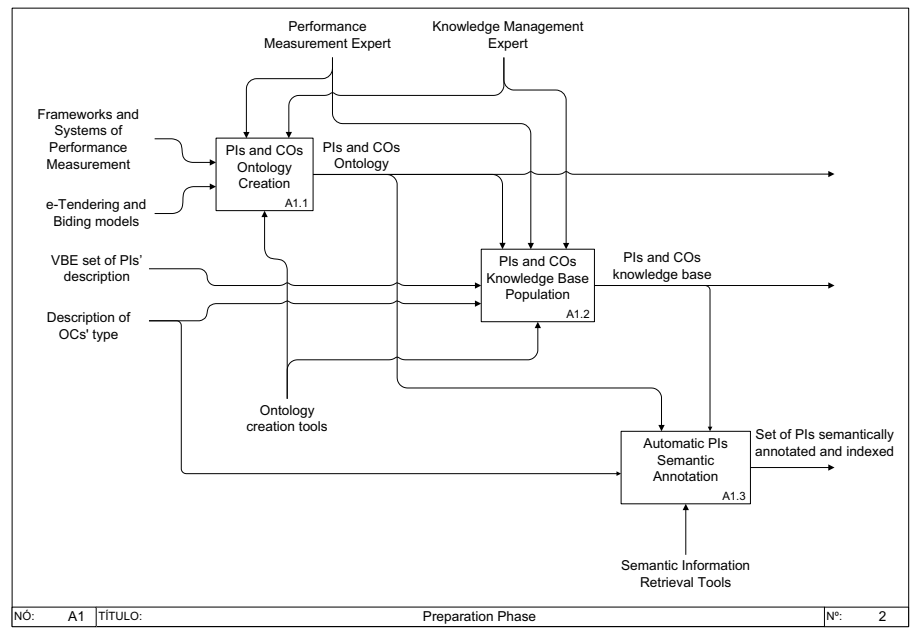

Figure 2. IDEF0 of Preparation Phase 


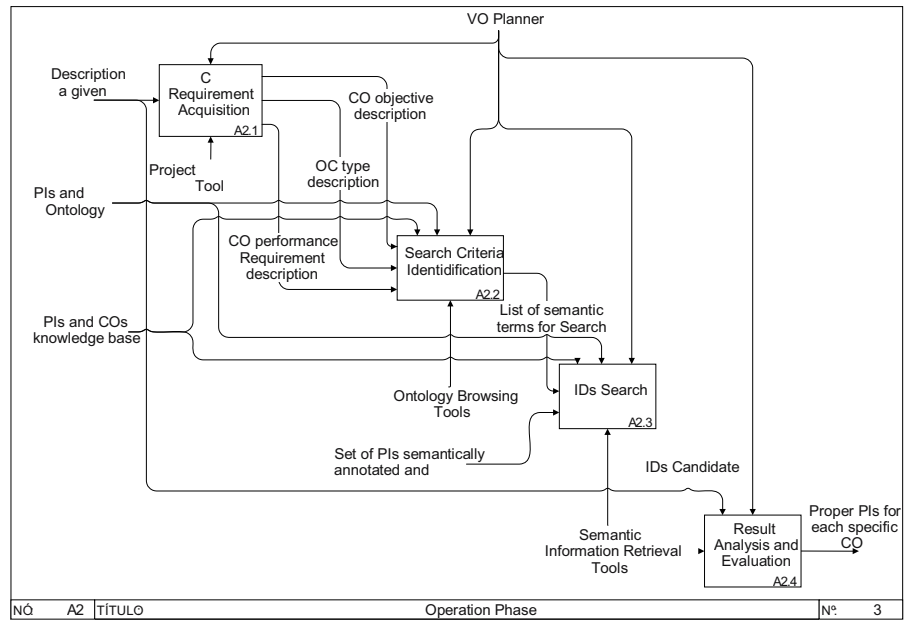

Figure 3. IDEF0 of Operation Phase

The very final output of the whole process is the set of proper PIs suggested to search for VO partners for a given CO. This output can be further used as an input to the Partners Search and Suggestion macro process.

\section{PRELIMINARY RESULTS}

In order to realize if the PIs selection process modeling works and provides better results against other methods, like SQL-based information retrieval, a preliminary case study has been conducted. A pilot from the ECOLEAD ${ }^{4}$ project was selected and a certain $\mathrm{CO}$ was chosen. More than two hundred PIs were populated in the VBE's database. Having both $\mathrm{CO}$ description and PIs, some PIs selection performance estimation was realized regarding some classic metrics used to measure performance in information retrieval systems, which are precision and recall. The former is calculated through the division of relevant documents retrieved by the total of retrieved documents, and the latter is calculated through the division of relevant retrieved documents by the total of relevant documents (Baeza-Yates and Ribeiro-Neto, 1999).

The addition of semantics in non-structured textual information is supported by the semantic annotation technique, as stated in section 2. Therefore, in order to semantically annotate the PIs' description it is necessary to specify a comprehensive ontology that describes all the relevant concepts related to PIs' characterization in a sensible way. A preliminary version of such ontology is presented in Baldo et al. (2007).

About the $\mathrm{CO}$ description, the information structure exemplified below follows the basic template for CO Description and Characterization proposed in ECOLEAD (Demsar et al., (2007). This template is composed of about 200 attributes, varying from general aspects (like $\mathrm{CO}$ id, objectives and requirements) to more detailed ones (like work breakdown structure, required competencies and resources). A partial instance of this $\mathrm{CO}$ template is showed below. 
Product Name: Centrifuge Machine

Objectives: Design and manufacturing of a centrifuge machine to separate various components of blood.

Sector. Health sector

Type: Collaborative Project Modality

Specific Requirements:

- Aerodynamic compact construction for vibration free performance;

- Power rotor to efficient separate micro substances;

- Body made of strong fabricated \& corrosion resistant steel;

- Speed range 100 to $6000 \mathrm{rpm}$ and above, accuracy $1 \mathrm{rpm}$;

- Capable to operate continuously in a temperature of $10-40{ }^{\circ} \mathrm{C}$ and relative humidity up to $80 \%$.

It can be noted that the main focus of this $\mathrm{CO}$ is quality, addressed by terms like efficient, accuracy, strong and resistant. Therefore, a possible complete target semantic query to retrieve PIs concerning this specific CO could be: "Look for PIs that measure production, with the objective of accuracy, considering the perspective of quality, in the domain of manufacturing industry". It is important to mention that the construction of semantic queries is driven by the human user and it is supported by a tool developed for that. Figure 4 shows a screenshot of this tool, with an example of the query and the results found out.

For comparison purposes, the semantic-based target query was decomposed into four pieces, from the smallest one ("Look for PIs that measure production") to the largest one (the whole semantic target query). These four semantic-based queries were compared against four correspondent SQL-based queries formed with the same terms as those used in the semantic ones. For instance, the semantic-based query "Look for PIs that measure production" has a correspondent SQL-based query like "select * from PIs where PI_description like "\%production\%". This strategy was adopted in order to show that the higher the number of search terms is the better the results provided by semantic queries are, regarding other information retrieval methods. These performance tests have been performed in a set of 237 PIs where 12 were identified as relevant ones by experts, previously.

Figure 5 presents the precision and recall performance results of both semantic-based and SQL-based queries. Axis $\mathrm{X}$ represents each one of the four queries and axis $\mathrm{Y}$ the percentage of precision and recall in a) and b), respectively. In the execution of the fourth query, the semantic one retrieved 15 PIs, where 3 were not relevant $(80 \%$ of precision and $100 \%$ of Recall). As can be seen in Figure 4, "Failure rate based on acceptation" is an example of no relevant PI that was retrieved by semantic queries. On the other hand, SQL-based query retrieved 9 PIs, where just 7 were relevant ( $77 \%$ of precision and $58 \%$ ). 


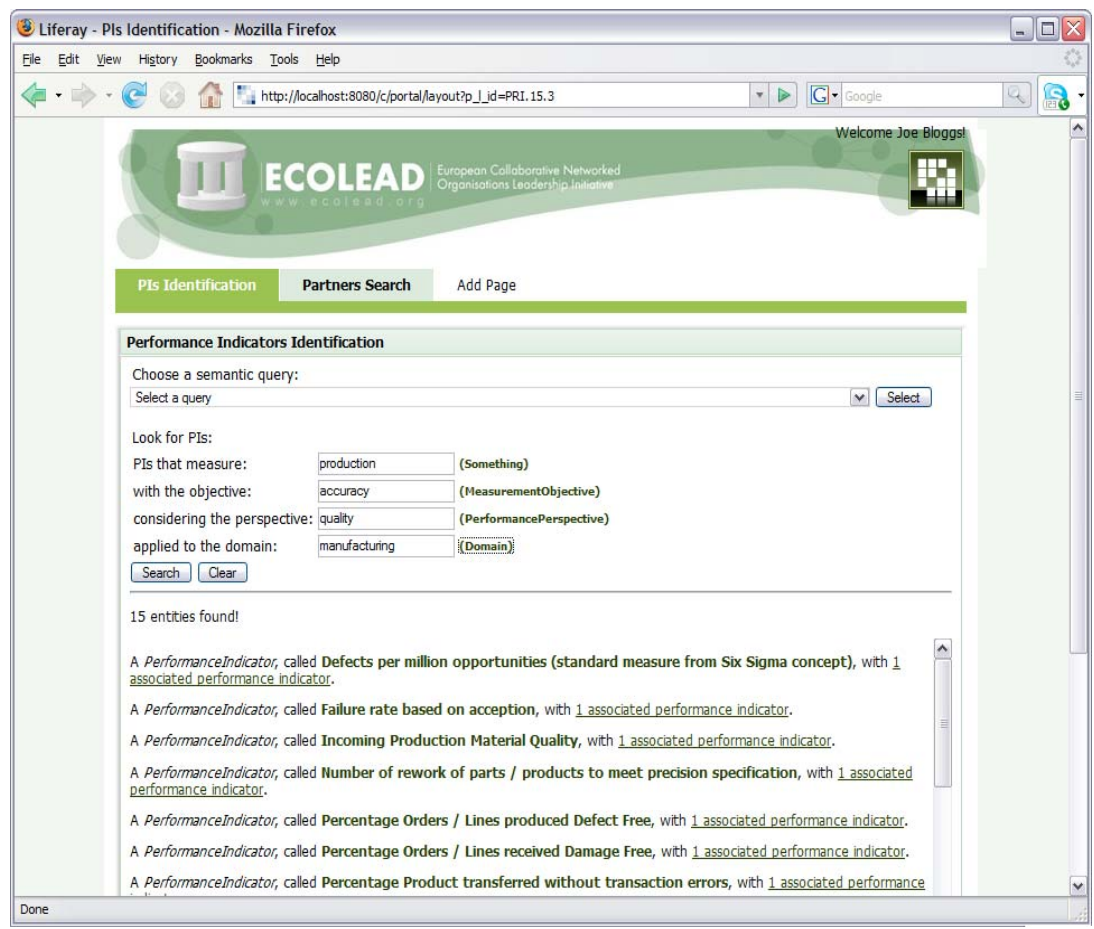

Figure 4. Screenshot of PIs Selection Tool

As the result, it can be noted that semantic queries had a slight higher precision because they have retrieved all the relevant PIs in spite of having retrieved few nonrelevant PIs. On the other hand, the results achieved via SQL didn't bring even the relevant PIs. Anyway, the final decision about which PIs will be indeed used in the partners' search is taken by the human manager, who is then assisted in this process.

Considering recall, again semantic queries had a higher performance due to their ability to deal with synonymous. For instance, when considering accuracy, the PIs related to precision (correctness and error, detect or damage free) also would be retrieved. Figure 4 presents some examples of retrieved PIs that have accuracy synonymous like, "Defect free", "Damage free" and "Without error". This is something that most of the other information retrieval techniques don't do.

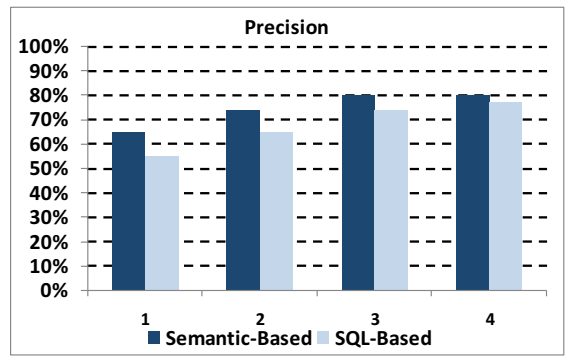

a) precision performance

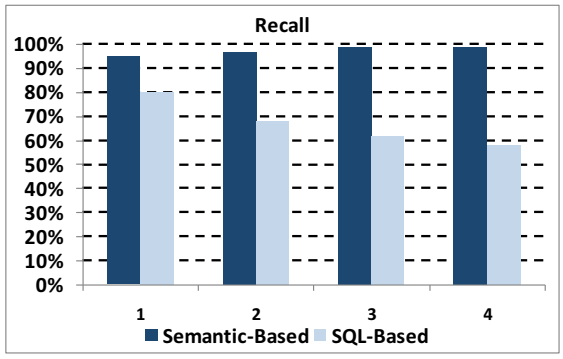

b) recall performance

Figure 5. Performance of Semantic-based queries against SQL-based ones 


\section{CONCLUSION}

This work has presented a contribution to the problem related to the criteria selection to suggest VO partners. To this end, the problem has been formalized and the involved processes modeled. This modeling aims at improving the user's decision concerning the selection of organizations, giving additional information about what can be used to compare the possible VO partners in a more effective way, as well as to systemize the whole process. The user can have different criteria to compare candidates for different COs. Thus, it is possible to find better partners comparing them via PIs that better represent the performance expected to this VO. Some preliminary tests have been made in the ECOLEAD project. First results showed that this model can work properly for, at least, the manufacturing industry sector (as most of the ECOLEAD partners belong to this sector).

It is an ongoing work. Although the whole model and software are already developed, validation activities will now be the focus of the work. It comprehends activities of measuring the effective usefulness of the retrieved PIs against the CO's specifications and VO's specificities. At another level, next activities consist in the deployment of the system at some ECOLEAD project's pilots and the application of the methodology in a real case scenario.

\subsection{Acknowledgements}

This work has been supported by CAPES Brazilian Research Agency and European Commission. It has been developed in the scope of the European IST ECOLEAD (www.ecolead.org) and of the Brazilian IFM II projects (www.ifm.org.br).

\footnotetext{
1 VBE (Virtual organization Breeding Environment) represents a long-term association of organizations prepared to cooperate and to establish VOs in the right time COs are identified (Afsarmanesh et al. 2005).

${ }^{1}$ SCOR (Supply-Chain Operations Reference-Model) is a reference model for supply-chain management systems and practices (www.supply-chain.org).

${ }^{1} \mathrm{http}: / /$ www.idef.com/idef0.html

${ }^{1}$ European Collaborative networked Organizations LEADership initiative Project aims to create strong foundations and mechanisms needed to establish advanced collaborative and network-based industry society in Europe (www.ecolead.org).
} 


\section{REFERENCES}

1. Afsarmanesh, H.; Camarinha-Matos, L.M. "A Framework for Management of Virtual Organization Breeding Environments". In Sixth IFIP Working Conference on Virtual Enterprises. Valencia, Spain: Springer, 2005.

2. Baeza-Yates, R.; Ribeiro-Neto, B., Modern information retrieval. England: Addison-Wesley Harlow, 1999.

3. Baldo, F.; Rabelo, R.J.; Vallejos, R.V. "An Ontology-Based Approach for Selecting Performance Indicators for Partners Suggestion". In Eighth IFIP Working Conference on Virtual Enterprises. Guimarães, Portugal: Springer, 2007.

4. Bittencourt, F.; Rabelo, R.J. "A Systematic Approach for VE Partners Selection Using the SCOR Model and the AHP Method". In Sixth IFIP Working Conference on Virtual Enterprises. Valencia, Spain: Springer, 2005 .

5. Camarinha-Matos, L.M.; Afsarmanesh, H.; OLLUS, M. "ECOLEAD: A Holistic Approach to Creation and Management of Dynamic Virtual Organization". In Sixth IFIP Working Conference on Virtual Enterprises. Valencia, Spain, 2005

6. Crispim, J.A.; Sousa, J.P. "Multiple Criteria Partner Selection in Virtual Enterprises". In Eighth IFIP Working Conference on Virtual Enterprises. Guimarães, Portugal: Spring, 2007

7. Demsar, D.; Mozetic, I.; Lavrac, N. "Collaboration Opportunity Finder". In Eighth IFIP Working Conference on Virtual Enterprises. Guimarães, Portugal: Spring, 2007

8. Dorado, A.; Izquierdo, E. "An Approach for Supervised Semantic Annotation". In Workshop on Image Analysis for Multimedia Interactive Services. 2003.

9. Grudzewski, W.M.; Sankowska, A.; Wantuchowicz, M. "Virtual Scorecard as a Decision-making Tool in Creating Virtual Organisation". In Sixth IFIP Working Conference on Virtual Enterprises. Valencia, Spain. 2005

10. Höbig, M., "Modellgestützte Bewertung der Kooperationsfähigkeit produzierender Unternehmen". In Fortschritt-Berichte VDI. Düsseldorf. 2002

11. Jarimo, T.; Pulkkinen, U. "A Multi-Criteria Mathematical Programming Model for Agile Virtual Organization Creation". In Sixth IFIP Working Conference on Virtual Enterprises. Valencia, Spain: Spring. 2005

12. Kiryakov, A.; Popov, B.; Terziev, I.; et al., "Semantic annotation, indexing, and retrieval". Web Semantics: Science, Services and Agents on the World Wide Web, 2004; 2(1): p. 49-79.

13. Petersen, S.A. "Using Competency Questions to Evaluate an Agent-based Model for Virtual Enterprises". In Fourth IFIP Working Conference on Virtual Enterprises. Lugano, Switzerland: Kluwer Academic Publishers. 2003

14. Rabelo, R.J.; Pereira-Klen, A.; Klen, E.R., "Effective Management of Dynamic Supply Chains". International Journal of Networking and Virtual Organizations, 2004.

15. Seifert, M.; Eschenbächer, J., "Predictive Performance Measurement in Virtual Organization". In Emerging Solutions for Future Manufacturing Systems, L.M. Camarinha-Matos, Editor. Springer. 2005, p. 299-307.

16. Vallejos, R.V.; Lima, C.P.; Varvakis, G. A Framework to Create a Virtual Breeding Environment in the Mould and Die Sector. in Seventh IFIP Working Conference on Virtual Enterprises. 2006. Helsinki, Finland.

17. Westphal, I.; Thoben, K.D.; Seifert, M. Measuring Collaboration Performance in Virtual Organizations. in Eighth IFIP Working Conference on Virtual Enterprises. 2007. Guimarães, Portugal: Springer. 